\title{
La tabla de eclipses en el códice de Dresden. Más de cien años de estudio y discusión
}

\author{
The Dresden codex eclipse table. More than one hundred years of study and discussion
}

\author{
David Marín*, Joshua Lemus \\ Escuela de Ciencias Físicas y Matemáticas, Universidad de San Carlos de Guatemala, Guatemala.
}

*Autor al que se dirige la correspondencia: marinroma@fisica.usac.edu.gt

Recibido: 02 de junio 2018 / Revisión: 28 de agosto 2018 / Aceptado: 25 de enero 2019

\section{Resumen}

$\mathrm{E}$ 1 códice Dresden es el más antiguo de tres libros escritos con glifos mayas que han sobrevivido hasta nuestros días. Contiene tablas y almanaques relacionados a los planetas visibles y una tabla con 69 intervalos de $177 \mathrm{o}$ 148 días relacionados a eclipses de sol y de luna que a pesar de haber sido estudiada por más de 100 años, sigue motivando ideas y propuestas sobre su posible uso y significado. La opinión más aceptada es que la tabla es un instrumento para predecir la posibilidad de eclipses, sin embargo algunos investigadores piensan que puede ser un registro de eclipses observados si se cambia la correlación entre los calendarios maya y gregoriano, lo cual ha llevado a proponer otras correlaciones entre calendarios. Algunos artículos proponen que la tabla se refiere a eclipses de sol, otros a eclipses de luna, o incluso de ambos tipos. El objetivo del presente estudio es comparar los datos en la tabla de eclipses con el catálogo de eclipses ocurridos del año cero al año 1500 de esta era y presentar bajo un mismo instrumento las evidencias a favor de las varias hipótesis en la literatura científica sobre el tema, considerando solamente la información en las tablas numéricas. Aunque dejar de lado la información en los textos jeroglíficos e imágenes limita el alcance de las conclusiones, la intención es proporcionar una visión imparcial de los datos en la tabla que permita estimar la validez de los distintos argumentos propuestos en la literatura, como un primer paso para desarrollar instrumentos que sirvan para un estudio multidisciplinario enfocado en estudiar las diez figuras que sobresalen en la tabla, las cuales son tan elaboradas y contienen tanto detalle que es muy probable que correspondan a fenómenos que sí fueron observados por los antiguos astrónomos.

\section{Abstract}

$\mathrm{T}$ The Dresden codex is the oldest of three Maya hieroglyph books that survived to this day. It contains tables and almanacs related to the visible planets and a table with 69 intervals of 177 and 148 days related to lunar and solar eclipses that in spite of being studied for more than 100 years, it continues to motivate ideas and proposals about its possible use and meaning. The most accepted opinion is that the table is an instrument to predict the possibility of eclipses, nonetheless some researchers think that it can be a register of observed eclipses if the correlation between Mayan and Gregorian calendars is changed, and this led to new proposals for the correlation between calendars. Some articles argue that the table refers to solar eclipses, others propose lunar eclipses, or both. The present study compares the data in the eclipse table to the catalogue of all eclipses on earth from the year zero to $1500 \mathrm{CE}$, and presents under the same instrument the evidences in favor of different hypothesis in the scientific literature on this topic, considering only the information in the numerical tables. Although leaving aside the information in the hieroglyph texts and images limits the scope of the conclusions, the intention is to present an impartial view of the data in the table to estimate the validity of the various arguments proposed in the literature, as a first step in developing instruments to be used on a multidisciplinary study of the ten outstanding figures in the table, which are so elaborated and contain so much detail that it is quite likely that they correspond to events actually observed by the ancient astronomers. 


\section{Introducción}

El códice de Dresden consta de 74 páginas de 9.1 x $20.5 \mathrm{~cm}$ de alto. Las hojas, pintadas de ambos lados están hechas de corteza de amate (árbol del género Ficus) cubiertas de una fina capa de estuco. Las pruebas de carbono 14 hechas a este material combinadas con un análisis iconográfico y etnográfico apuntan a Yucatán como lugar de origen, alrededor del siglo XIII o XIV (Satterthwaite \& Ralph, 1960). Sin embargo algunos investigadores piensan que el material que incluye el códice fue recopilado a lo largo de cientos de años, pasando de una generación a otra, por lo que el contenido del mismo sería mucho más antiguo (Milbradth, 1999). Hay 27 fechas escritas en el códice, la más antigua es del año 623 y la más reciente corresponde al año 1210 según la correlación GMT (Goodman-Martínez-Thompson) que es la más aceptada entre los calendarios maya y juliano usado en Europa hasta 1582.

Se piensa que fue llevado a Europa en el siglo XVI y permaneció olvidado por unos 200 años. En 1739 el director de la Biblioteca de Dresden lo compró a un coleccionista y pasaron más de 160 años hasta que la primera versión con las páginas en orden correcto y comentada fue publicada por Förstemann (1906), lo cual abrió las puertas a otros investigadores a estudiar el documento. Por motivos históricos la numeración de las páginas del códice que más se usa en la literatura es la numeración incorrecta de una versión anterior.

Desde un inicio Förstemann notó que las páginas 51 a 58 contienen una tabla que empieza en la mitad superior de la página 51 y continúa hasta la mitad superior de la página 58 (registro a), pasando luego a la mitad inferior de la página 51 y terminando en la mitad inferior de la página 58 (registro b). Él dedujo que la tabla se refería a la luna ya que de la página 53(a) a la 58(b) hay 69 columnas de glifos que terminan con los números 177 y 148 , que son muy cercanos a número de días que hay en seis y cinco lunaciones respectivamente. Insertadas entre las 69 columnas de glifos hay diez figuras que tienen en la parte superior dos columnas de textos jeroglíficos más elaborados. En 1913 Meinshausen fue el primero en publicar que la tabla en las páginas 51 a 58 se refiere a eclipses debido a que sumando el número de días que hay entre una figura y otra se obtienen períodos de eclipses como los que serían observables desde una localidad cualquiera en el planeta. Él identificó los glifos correspondientes a eclipses de sol y de luna, y notó que la aparición de éstos en pares podía significar que las figuras se refieren a eclipses de luna y sol visibles desde la misma localidad. La relación de la tabla con eclipses fue descubierta independientemente casi al mismo tiempo por Willson, pero sus estudios no fueron publicados hasta 1924 por Spinden. Willson notó que el largo de la tabla $(11,960$ días) es muy cercano a 69 veces el período promedio entre estaciones de eclipses (173.31 días), además los intervalos entre las diez figuras lo llevaron a suponer que éstas correspondían a un registro de eclipses observados. Él mismo puso a prueba esta hipótesis comparando los intervalos entre figuras con el catálogo de eclipses sucedidos en el período del año -12 al año 1520 de la presente era, publicado por Oppolzer en 1887, sin embargo no encontró ninguna serie de eclipses visibles desde Yucatán que correspondiera con los intervalos entre las figuras (Aveni, 2001). Este estudio sentó la base de la opinión más aceptada hasta hoy: la tabla es un instrumento para anticipar la posibilidad de que suceda un eclipse iniciando el conteo en una fecha conveniente.

Las diez figuras intercaladas entre los 69 intervalos se conocen como "eclipse warning stations" ("estaciones de advertencia de eclipses") indicando que ese número de días a partir de la fecha inicial de la tabla se espera que suceda un eclipse.

El posible uso de la tabla de eclipses también ha sido objeto de numerosos estudios. En 1931 Teeple notó que tres estaciones de eclipses $3 \times 173.31 z=519.93$ son casi dos períodos del calendario tzolk' in de 260 días (Thompson, 1972), de manera que los eclipses pueden darse sólo en tres regiones del ciclo de 2x260 días, habiendo fechas en las que no se ven eclipses, aunque haya luna nueva o luna llena.

En las primeras dos páginas de la tabla aparecen múltiplos y casi múltiplos del largo total de la tabla, esto ha sido interpretado por varios investigadores como un indicio de que la tabla era reciclada varias veces cubriendo períodos más largos. Uno de los estudios más completos del posible uso de la tabla aparece en Bricker y Bricker (2011), ellos consideran que la tabla pudo ser usada varias veces usando algún mecanismo de corrección similar al usado en la tabla de Venus del mismo códice. Sin embargo hay múltiplos de 31 y 39 veces el largo de la tabla y es difícil que la tabla pueda ser usada con éxito por períodos tan largos.

Lo que debió ser un trabajo formidable para los primeros investigadores se vuelve cada vez más accesible con el uso de computadoras y modelos matemáticos para simular el cielo que se han ido perfeccionando al pasar los años, lo cual justifica revisar los cálculos para comprender el alcance y significado de estos estudios. 
Otra motivación para el presente estudio es que no todos han llegado a las mismas conclusiones. Dependiendo de las preferencias y suposiciones hechas es posible encontrar evidencias a favor de distintas hipótesis.

En la actualidad la mayoría de investigadores consideran que la correlación entre calendarios maya y juliano GMT está bien establecida y desarrollan su interpretación con esta base, interpretación que se ve complicada por el hecho de que usando esta correlación el registro de eclipses de la tabla no corresponde con eclipses visibles desde el área ocupada por la civilización maya. Otros opinan que el hecho de que la tabla de eclipses no corresponda con eclipses observables desde Yucatán es motivo para dudar de la correlación entre calendarios, y buscan series de eclipses de sol o de luna a lo largo de la historia que correspondan con la tabla, y proponen nuevas correlaciones en base a estas observaciones (Macri \& Vail, 2009).

Aunque la correlación GMT se basa en sólidos argumentos etno-históricos, se pueden imaginar razones para que distintos pueblos mesoamericanos llevaran fechas distintas producto de errores, omisiones, discrepancias políticas o filosóficas, aislamiento o alguna otra razón (por ejemplo que la tabla esté "encriptada" y sólo los iniciados sepan qué número agregar a la fecha inicial). Después de todo, se sabe que el códice fue escrito alrededor del siglo XIII y hace referencias a eventos sucedidos en el siglo VIII, es probable que algo haya sucedido en ese tiempo que obscurezca la fecha inicial. De hecho una de las cuatro fechas iniciales de la tabla contiene errores que no se pueden conciliar, y en la práctica se deja de lado para no complicar más las cosas.

Por estos motivos es válido investigar si hay series de eclipses observables desde la región ocupada por la civilización maya que coincidan con la secuencia de la tabla de eclipses en el códice, y reunir en un mismo estudio las evidencias en favor de las posibilidades que surgen en respuesta a las interrogantes: ¿son eclipses de sol o de luna? ¿Es un registro de eclipses observados o un instrumento para predecir eclipses? tomando en cuenta que estas opciones no son necesariamente excluyentes.

El punto de mayor interés para nuevos estudios de la tabla de eclipses son las diez figuras que se encuentran intercaladas entre los 69 intervalos de 177 y 148 días. Si la tabla del códice fuera solamente un instrumento para predecir posibles eclipses, estas figuras no están puestas en el mejor lugar posible (estadísticamente), sin embargo el número de días transcurridos entre una figura y otra sí corresponden a períodos entre eclipses observables desde una localidad particular en el planeta (como puede verse consultando un catálogo de eclipses). Además, son tan elaboradas y contienen tanto detalle que todavía no ha sido del todo esclarecido por los estudios de epigrafía, que cuesta mucho creer que son adornos que no dicen nada sobre cómo se vio el cielo en esas fechas.

\section{Materiales y métodos}

Para estudiar la coincidencia de tabla de eclipses en el códice con el catálogo de eclipses de sol o de luna ocurridos durante el intervalo del año cero al año 1500 se genera una serie de fechas de presuntos eclipses sumando los 69 intervalos que hay en la tabla a una fecha inicial. Las fechas en el códice están dadas en el sistema llamado "cuenta larga" que es el número de días transcurridos desde el comienzo de una era. Según la correlación GMT el inicio de la cuenta larga sería el 11 de agosto del año $3114 \mathrm{AC}$ en el calendario gregoriano. Este sistema es semejante al sistema llamado la cuenta de días julianos usado actualmente en astronomía. Este sistema usa el número de días transcurridos desde una fecha inicial que corresponde al 24 de noviembre del 4714 AC (calendario gregoriano). La relación entre la cuenta larga y el número de días julianos se obtiene sumando una "constante de correlación" al número en la cuenta larga para obtener el número de día juliano.

La fecha inicial de la tabla en la cuenta larga es: $1,412,848$ días. Sumando la constante de correlación GMT $(584,283)$ se obtiene el número de día juliano 1,997,131 que corresponde al 10 de noviembre del 755 (en el calendario gregoriano).

Para obtener una estadística de qué tanto coincide la tabla de eclipses con el registro de eclipses ocurridos hacemos coincidir la fecha inicial de la tabla con el primer eclipse ocurrido en el año cero de esta era y notamos las coincidencias en la serie de eclipses generada sumando los 69 intervalos a esta fecha inicial. Luego hacemos coincidir la fecha inicial del códice con la fecha del segundo eclipse sucedido en el año cero y repetimos el procedimiento, y así sucesivamente repitiendo el procedimiento con cada eclipse sucedido hasta llegar al último eclipse en el 1500 de esta era. Esto equivale a variar la constante de correlación desde un valor de 308,211 hasta un valor de 856,075, es decir una variación de 1,500 años.

El objetivo de variar tanto la constante de correlación es averiguar cuántas series de eclipses han suce- 
dido que coinciden con los datos de la tabla (es decir, qué tan buen instrumento de predicción es). Para ello se define la variable "coincidencia exacta", que aumenta en una unidad por cada fecha en la serie generada que también se encuentra en el catálogo de eclipses, y se define la variable "coincidencia" que aumenta en una unidad por cada fecha en la serie generada que se encuentra dentro de un intervalo de \pm 1 día de una fecha en el catálogo de eclipses. Esta ventana de \pm 1 día es motivada por las fechas en el códice, dadas como tres días consecutivos en el calendario. Primero se consideran como fechas iniciales las que aparecen escritas en el códice, según la correlación GMT (y GMT +2). Luego se repite esta operación tomando como fecha inicial todas las fechas de eclipses en el catálogo de eclipses de sol y de luna sucedidos en el período del año cero al 1500, con lo cual se obtiene una distribución para la variable coincidencia a nivel global (Figura 2).

Algunos investigadores (Bricker \& Bricker, 2011) consideran que la aparición de al menos tres fechas iniciales separadas por 15 días sugiere que la ventana para una posible estación de eclipses debe considerarse como 30 días. Hay que notar que con una ventana tan grande, y considerando cualquier eclipse, sea visible o no en el área maya, la tabla no falla en predecir eclipses que sí suceden, aunque predice eclipses que no suceden.

El procedimiento se repite para los catálogos de eclipses visibles desde varias localidades en Mesoamérica: Copán, Chichén Itzá, Palenque, Piedras Negras, Quiriguá, Tikal, Yaxchilán y La Venta. La principal diferencia es que un eclipse que es total o anular en una ciudad, puede que sea solo parcial o no visible en otra. Para las tablas y gráficas que ilustran este informe se toma una posición central en el área de estudio: $18^{\circ}$ latitud Norte, $90^{\circ} 301$ longitud Oeste (cerca del sitio arqueológico La Misteriosa en Campeche), y algunas variantes según la posición se mencionan en la discusión de resultados.

Finalmente se repite el procedimiento anterior tomando sólo los intervalos entre las 10 figuras y comparándolos con el catálogo de eclipses visibles desde la región ocupada por la civilización maya. La idea es estudiar la posibilidad de que las 10 figuras correspondan con fenómenos observables desde alguna ciudad maya.

\section{Catálogo de eclipses de sol y luna}

El presente estudio utiliza los catálogos de eclipses producidos por Espenak y Meeus $(2006 ; 2009)$ publicados por la Agencia Espacial Estadounidense
(NASA) que contienen tablas con información sobre eclipses de sol y de luna calculados en un período de 5,000 años (desde el -1999 hasta el 3000), el cual está disponible en el sitio web de la NASA, donde también se encuentra la aplicación: Javascript solar(lunar) eclipse explorer, que permite generar catálogos de eclipses visibles desde una localidad en particular, y fue usado para generar los datos de eclipses visibles desde varias ciudades mayas del período clásico.

Durante el período estudiado hubo 3,612 eclipses de luna y 3,541 eclipses de sol. Considerando solo los eclipses visibles desde la posición $18^{\circ}$ latitud Norte, $90^{\circ}$ 301 longitud Oeste tenemos 540 eclipses de sol y 1,638 eclipses de luna visibles en el mismo período.

Los catálogos de Espenak y Meeus fueron calculados usando la teoría de variaciones seculares en las órbitas planetarias, conocida como VSOP87. La primera versión fue publicada por Bretagnon (1982). La posición de la luna es calculada usando la teoría ELP 2000/82 de Chapront-Touzé y Chapront (1983). Para modelar el tamaño y la forma de la sombra de la luna se usa el método de Danjon (1951). Las incertezas en estos modelos son mucho menores a la incerteza causada por el hecho de que la velocidad de rotación de la tierra cambia debido a fuerzas de marea causadas por la luna.

El tiempo en los cálculos es una unidad uniforme TD (terrestrial dynamical time), mientras que las fechas en días se llaman tiempo universal UT por sus siglas en inglés. El problema es que la duración del día va cambiando con el tiempo. Esta diferencia $\Delta \mathrm{T}=\mathrm{TD}$ - UT se conoce bien para épocas recientes, y existen modelos para épocas anteriores que son ajustados usando eclipses y otros fenómenos astronómicos registrados por distintas civilizaciones a lo largo de la historia. Esta es la principal causa de incerteza en determinar la visibilidad de un eclipse.

Además del catálogo de eclipses hemos usado el programa de código abierto Stellarium versión 0.13 que simula el cielo usando los mismos modelos VSOP87 y ELP82 y a partir de la versión 0.12 incluye también el modelo para calcular $\Delta \mathrm{T}$, lo cual permite comprobar los datos del catálogo y observar el cielo durante los eclipses estudiados. Ver la posición de las estrellas y planetas al momento de un eclipse es importante para tratar de asociar la iconografía en la tabla con eventos astronómicos. Esta información no la proporciona el catálogo de eclipses. 


\section{La tabla de eclipses en el códice de Dresden}

La tabla de eclipses ocupa las páginas 51 a la 58 del códice y cubre un intervalo de 11,960 días (32.75 años), y comprende 405 lunaciones (La numeración correcta es de la página 30 a la 37, pero en la literatura se quedó la numeración usada por los primeros investigadores). Cada página está dividida a la mitad por una línea horizontal de color rojo (en el original). La Figura 1, adaptada de la versión de Gates (1932) muestra las distintas secciones. La tabla se lee primero en la mitad de arriba de 51(a) a 58(a) y luego se pasa a la mitad de abajo de 51(b) a 58(b).

De la página 53(a) a la 58(b) hay 69 columnas (sin contar las 10 figuras), cada una empieza con un texto de dos glifos, luego un número en color negro que es un número de días acumulado hasta ese punto en la tabla, seguido de tres fechas consecutivas del tzolk' in y finalmente el número correspondiente a 177 o 148, que en notación maya se escriben (8.17) y (7.8) respectivamente. El total acumulado en una columna dada se obtiene sumando el total de la columna anterior al intervalo 177 o 148 de la columna en cuestión (algunas veces el número sumado es 178 aunque siempre se escribe 177).

En las páginas 51(a) y 52(a) hay siete columnas con múltiplos y quasi múltiplos del largo de la tabla $(\mathrm{L}=11,960)$ escritos en números negros y rojos entrelazados. Los números que aparecen son: $1 \mathrm{~L}+1820,2 \mathrm{~L}$, $3 \mathrm{~L}, 4 \mathrm{~L}, 5 \mathrm{~L}, 6 \mathrm{~L}+120,16 \mathrm{~L}, 17 \mathrm{~L}, 18 \mathrm{~L}, 31 \mathrm{~L}+260$ y $39 \mathrm{~L}$. Esto se ha interpretado como un indicio de que la tabla era reciclada varias veces (Aveni, 2001). El múltiplo 39L corresponde a 1,277 años.

Las últimas dos columnas de la página 52a contienen cuatro fechas en la cuenta larga, cada columna tiene dos fechas entrelazadas, una en rojo y otra en negro en el original (gris o negro en la Figura 1). En la última columna aparece en color negro la fecha (9.16.4.10.8) 12 lamat, el número en rojo es (9.16.4.11.3) 1 ak'abal (15 días después de 12 lamat), 15 días después se llega a la fecha escrita en negro (9.16.4.11.18) 3 etz'nab. La cuarta fecha en la página 52(a) no sigue este patrón y es probable que contenga errores de escritura (Bricker \& Bricker, 2011).

Según la correlación GMT, la fecha 12 lamat corresponde al 10 de noviembre del 755 (calendario gregoriano), o bien al 6 de noviembre del 755 en el calendario Juliano, en uso antes de 1582 y usado en las tablas de eclipses de Espenak y Meeus. Esta fecha corresponde al día juliano: 1997130.5 (el número de día juliano empieza al medio día de Europa, por eso la fracción de medio día)

\section{Intervalos en la tabla de eclipses}

Empezando en la página 53(a) y terminando en la página 58(b) hay 69 columnas que terminan en el número 177 o 148 , las columnas que terminan con el número 148 (7.8 en notación maya) siempre anteceden una figura. Hay 10 figuras intercaladas entre los 69 intervalos que tienen textos jeroglíficos más extensos en la parte superior (Figura 5). La Tabla 1 muestra los 69 intervalos de 177 y 148 días, así como el total de días entre cada figura. Estos totales no están escritos explícitamente en el códice, pero son intervalos que suceden entre eclipses visibles desde un mismo lugar, como puede verse en la Tabla 4 elaborada a partir del catálogo de eclipses visibles desde una localidad en Yucatán.

\section{Resultados}

\section{Coincidencias de 69 fechas con eclipses de sol y de luna, tomando como punto de partida fechas en el códice.}

En la Tabla 2 se muestra el número de coincidencias dentro del rango \pm 1 día de 69 fechas de presuntos eclipses con el catálogo de eclipses de luna y el de eclipses de sol, tomando como fecha inicial tres de las fechas que aparecen en la página 52(a) del códice: (9.16.4.10.8) 12 lamat, (9.16.4.11.3) 1 ak'abal, y (9.16.4.11.18) 3 etz'nab. La fecha en días julianos es según la correlación GMT. Estas fechas están separadas por 15 días. Es importante notar que no hay ninguna correlación exacta en las series de eclipses generadas a partir de estas fechas con los eclipses en los catálogos.

\section{Coincidencias de 69 intervalos comparando con el registro de todos los eclipses de sol y de luna ocurridos del año cero al año 1500.}

En el período del año cero al 1500 de esta era hubo 3,612 eclipses de luna y 3,541 eclipses de sol. La Figura 2 muestra la distribución de coincidencias exactas y coincidencias dentro del rango de \pm 1 día de las series de fechas de eclipses generadas sumando los 


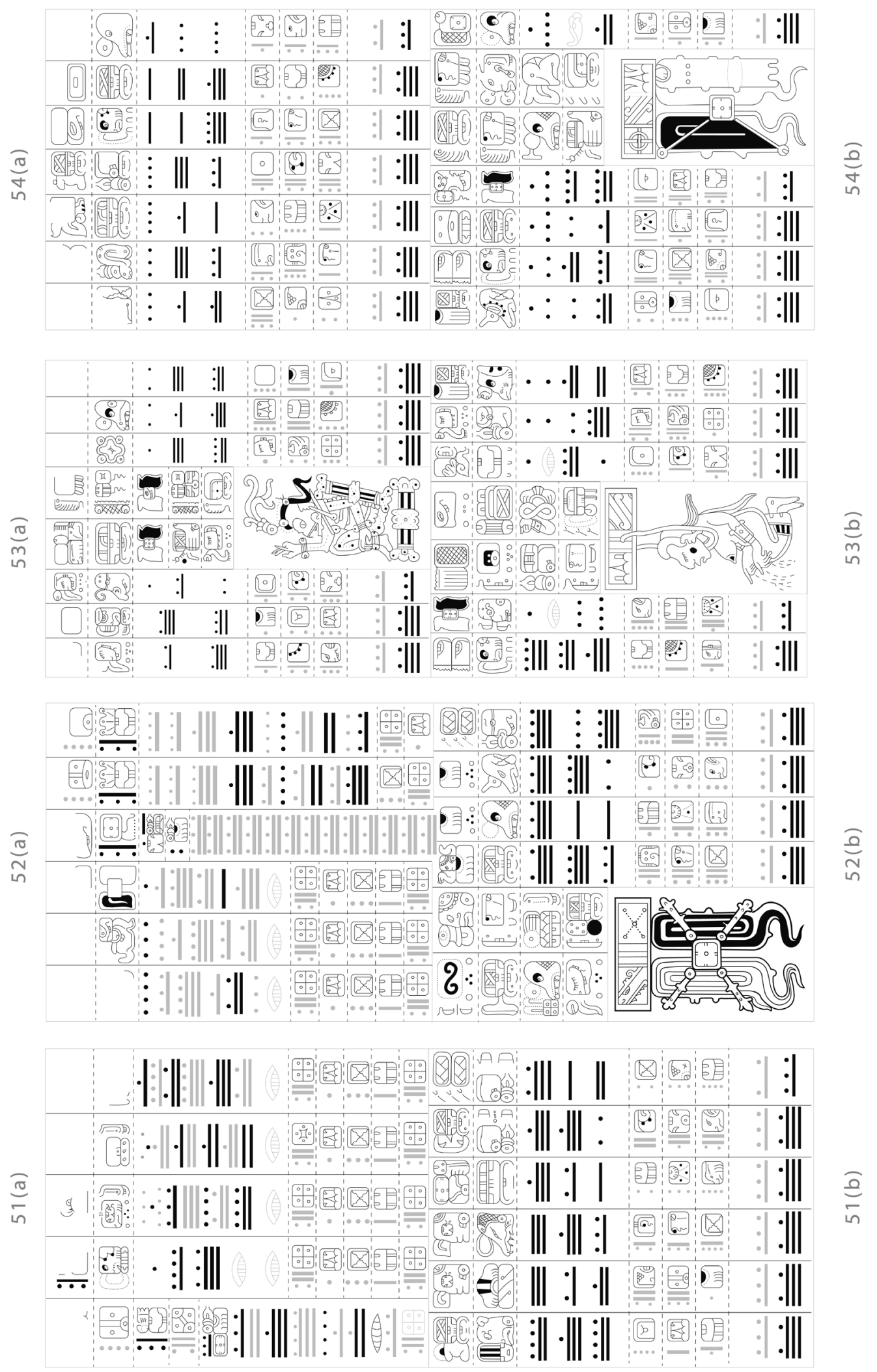



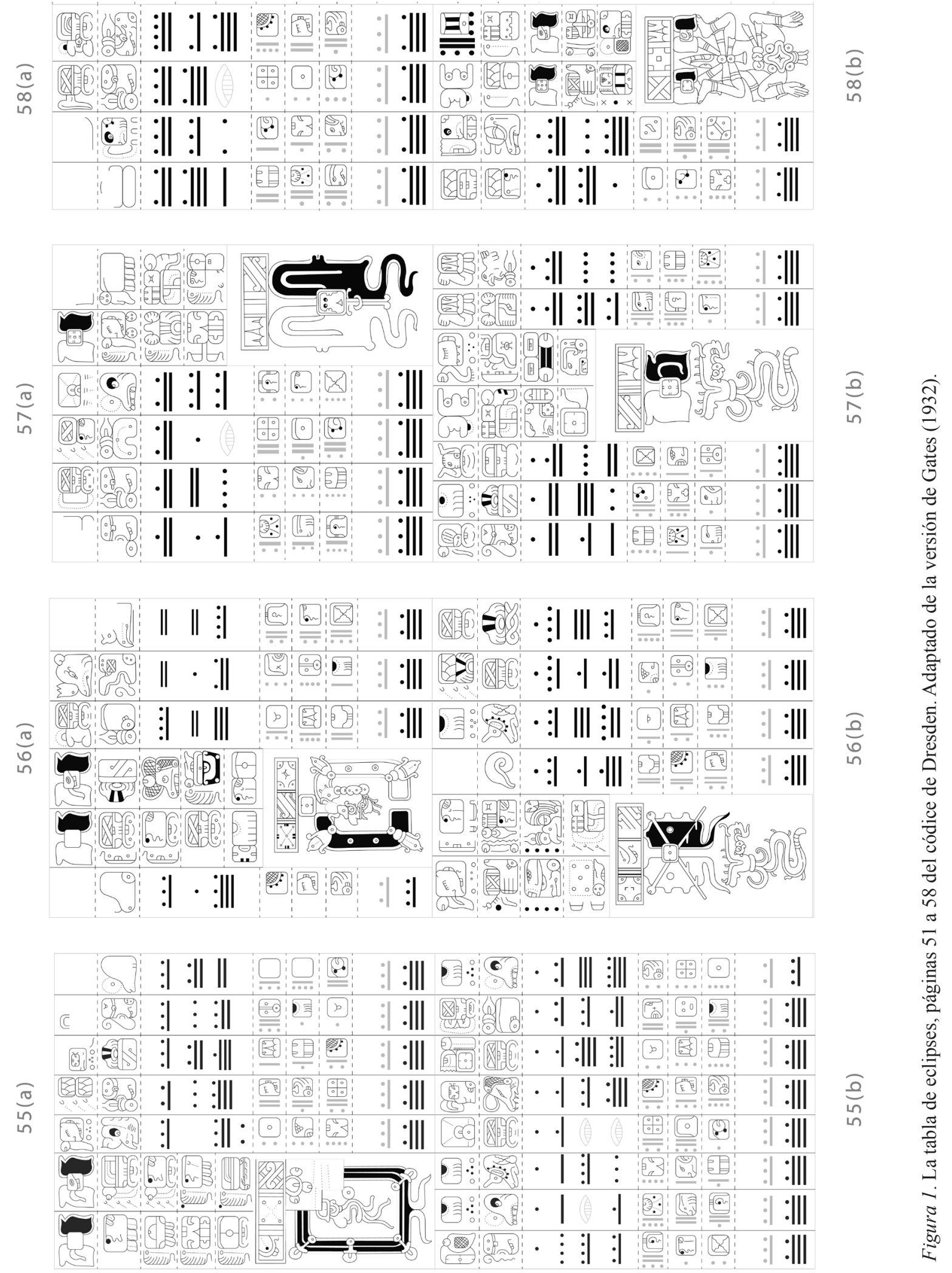


\section{Eclipses de luna}

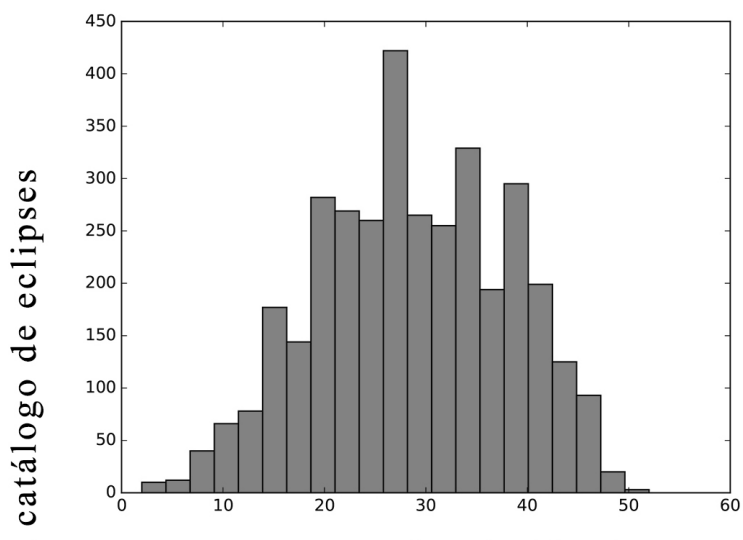

Eclipses de sol

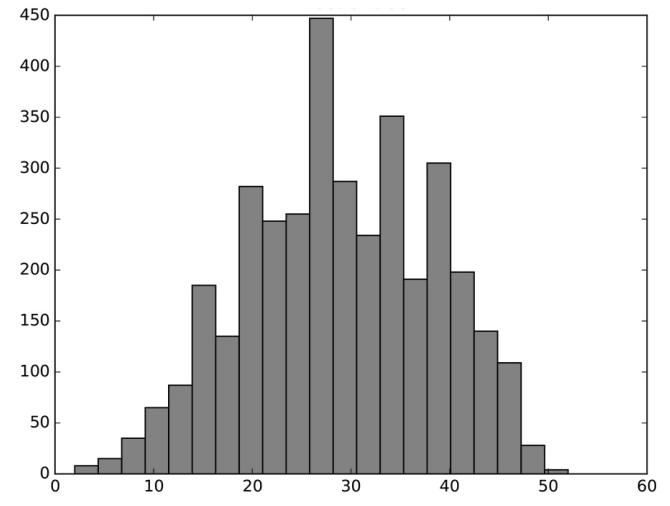

Número de coincidencias exactas de las series generadas a partir de los intervalos en el códice con el catálogo de eclipses de sol y luna.

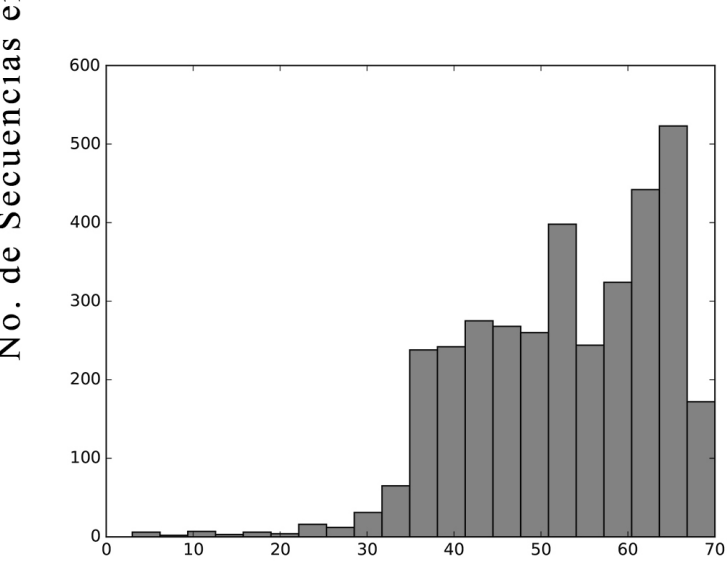

Número de coincidencias dentro de un intervalo de \pm 1 día

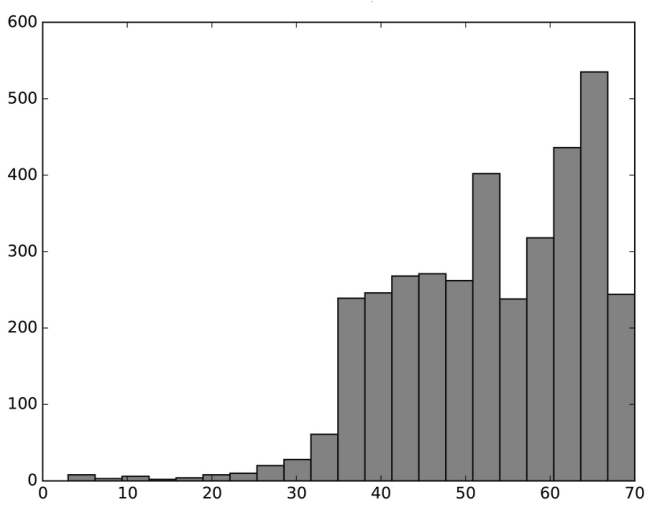

Figura 2. Coincidencias de 69 intervalos con el catálogo de eclipses de luna y eclipses de sol del año cero al 1500.

69 intervalos del códice a cada entrada de los catálogos de eclipses de sol y luna en el período estudiado.

\section{Coincidencias de 69 intervalos comparando con el catálogo de eclipses de sol y de luna visibles desde la localidad $18^{\circ}$ latitud Norte, $90^{\circ} 30$ í lon- gitud Oeste.}

Durante el período del año cero al 1500, el catálogo de eclipses de luna visibles desde esta localidad comprende 1,638 eclipses. El catálogo de eclipses de sol visibles desde la misma región tiene 540 eclipses. La Figura 3 muestra la distribución del número de coincidencias en las series de eclipses generadas con los catálogos de eclipses de sol y de luna vistos desde la posición $18^{\circ}$ latitud Norte, $90^{\circ} 30$ í longitud Oeste (sólo se muestran coincidencias dentro del intervalo de 1 día).

La mayor cantidad de coincidencias con eclipses de sol es una serie con 20 aciertos, una con 19 y dos con 18. La mayor cantidad de coincidencias con eclipses de luna son cuatro series con 42 coincidencias y 12 series con 41 . 
Tabla 1

Intervalos en la tabla de eclipses del códice

\begin{tabular}{lll}
\hline Intervalos (días) & Figura & Total (días) \\
\hline $177,177,148$ & $53 \mathrm{a}$ & 502 \\
$177,177,177,177^{*}, 177,177,177,177,177,148$ & $55 \mathrm{a}$ & 1,734 \\
$177^{*}, 177,177,177,177,148$ & $56 \mathrm{a}$ & 1,034 \\
$177,177,177,177^{*}, 177,177,148$ & $57 \mathrm{a}$ & 1,211 \\
$177,177,177^{*}, 177,177,177,177,177,177,148$ & $52 \mathrm{~b}$ & 1,742 \\
$177^{*}, 177,177,177,177,148$ & $53 \mathrm{~b}$ & 1,034 \\
$177,177,177,177,177,177,148$ & $54 \mathrm{~b}$ & 1,210 \\
$177,177,177^{*}, 177,177,177,177,177,148$ & $56 \mathrm{~b}$ & 1,565 \\
$177,177^{*}, 177,177,177,177,148$ & $57 \mathrm{~b}$ & 1,211 \\
$177,177,177,177$ & $58 \mathrm{~b}$ & 708
\end{tabular}

Nota. ${ }^{*}$ en ocasiones se suma 178 aunque el número anotado es 177

Tabla 2

Coincidencias de 69 intervalos en el códice con el catálogo de eclipses.

\begin{tabular}{lll}
\hline Fecha en días julianos & Coincidencias (Eclipses de luna) & Coincidencias (Eclipses de sol) \\
\hline 1997130.5 & 0 & 15 \\
1997145.5 & 43 & 0 \\
1997160.5 & 0 & 38 \\
\hline
\end{tabular}

\section{Coincidencias de fechas asociadas a las 10 figuras.}

La Figura 4 muestra la distribución del número de coincidencias en la serie de fechas correspondientes a los intervalos entre las diez figuras de la tabla de eclipses con los catálogos de eclipses de sol y luna visibles desde la posición $18^{\circ}$ latitud Norte, $90^{\circ} 30$ í longitud Oeste durante el período del año cero al año 1500.

Para los eclipses de luna observados desde la posición $18^{\circ}$ latitud Norte, $90^{\circ} 30$ í longitud Oeste, la mayor cantidad de coincidencias son 14 series con coincidencia de las diez fechas asociadas a figuras, y 98 series con nueve coincidencias.

Considerando solo los eclipses de sol visibles desde la posición $18^{\circ}$ latitud Norte, $90^{\circ} 30$ í longitud Oeste se encuentran ocho series con seis coincidencias de las 10 fechas asociadas a figuras. Estas series sucedieron en los años 50, 267, 350, 994, 1048, 11021142 y 1348.
La mayor cantidad de coincidencias encontrada es de siete coincidencias exactas en los eclipses observados desde las ciudades de Palenque, Piedras Negras y La Venta. Esta serie de siete coincidencias exactas inicia en el día juliano 2123848 que corresponde al 20 de octubre del 1102, muy cerca de la fecha en la que se piensa fue escrito el códice, pero que está casi 347 años más adelante que la fecha del año 755 dada en el inicio de la tabla.

\section{Intervalos entre eclipses, datos globales}

La Tabla 3 muestra los intervalos que suceden entre eclipses de luna y eclipses de sol a nivel global y el porcentaje de eclipses en cada rango para el período desde el año cero hasta el año 1500 en el que sucedieron 3,612 eclipses de luna y 3,541 eclipses de sol. 


\section{Eclipses de sol}

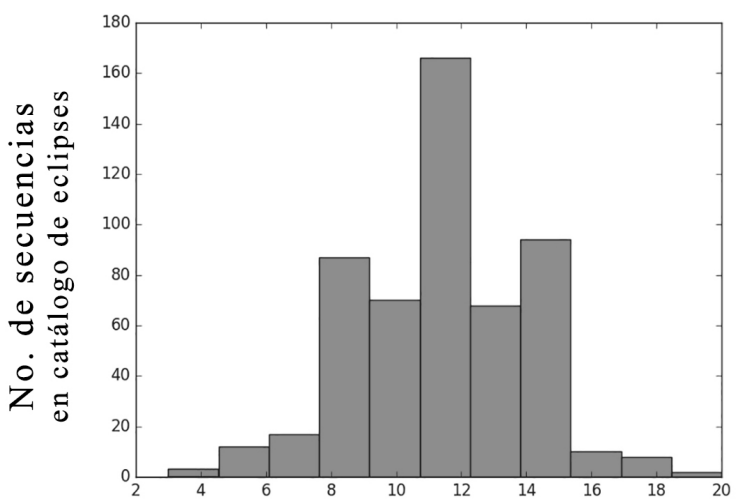

Eclipses de luna

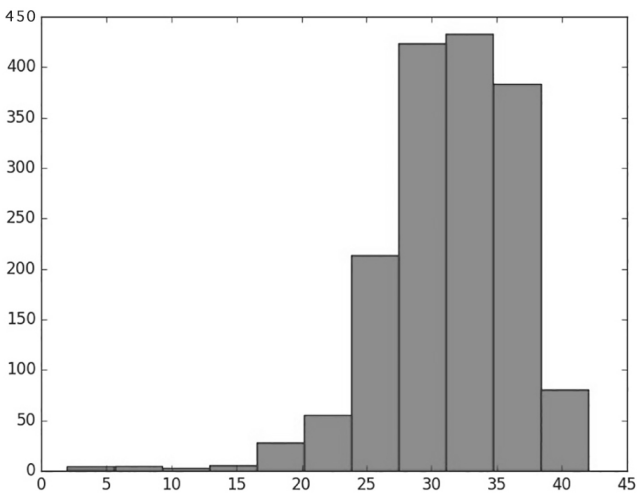

Número de Coincidencias dentro del intervalo de \pm 1 día en las series generadas a partir de los intervalos del códice con el catálogo de eclipses.

Figura 3. Coincidencias de 69 intervalos con los catálogos de eclipses de sol y luna visibles desde la posición

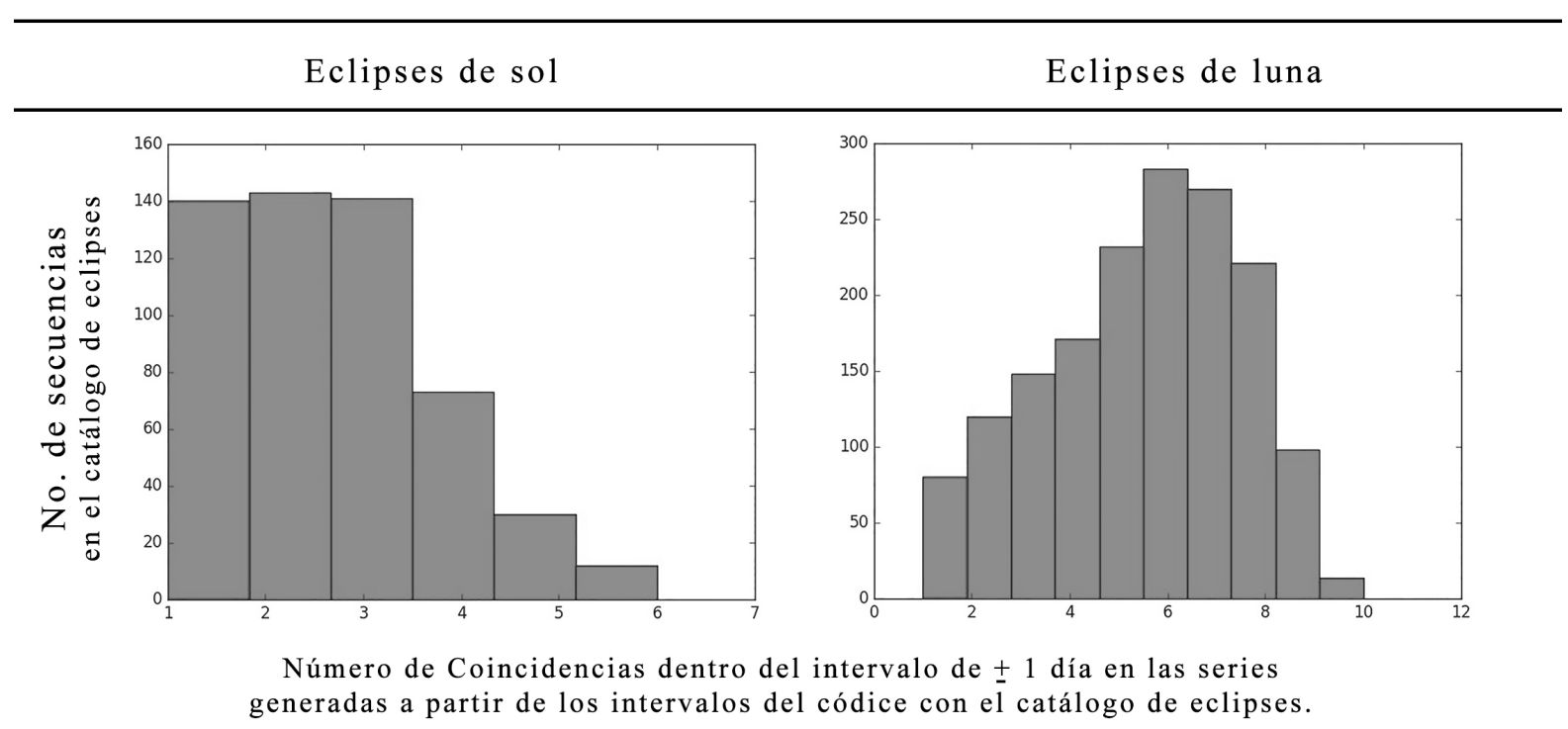

Figura 4. Coincidencias de fechas asociadas a las 10 figuras con eclipses de sol y luna vistos desde la posición $18^{\circ}$ latitud Norte, $90^{\circ} 301$ longitud Oeste. 
Tabla 3

Estadística de intervalos entre eclipses de sol y luna

\begin{tabular}{lll}
\hline Intervalo (días) & Lunar $(\%)$ & Solar $(\%)$ \\
\hline 29 & 6.56 & 5.9 \\
30 & 5.89 & 4.8 \\
146 & 0.33 & 0.39 \\
147 & 8.75 & 7.77 \\
148 & 11.71 & 11.15 \\
149 & 3.16 & 3.08 \\
176 & 12.07 & 12.62 \\
177 & 30.12 & 31.83 \\
178 & 20.4 & 21.4 \\
179 & 1 & 1 \\
\hline
\end{tabular}

Intervalos entre eclipses visibles desde la posición $18^{\circ}$ latitud Norte, $90^{\circ} 30$ í longitud Oeste.

La Tabla 4 muestra los intervalos entre eclipses de sol y luna y el porcentaje de eclipses sucedidos en cada intervalo, vistos desde la posición $18^{\circ}$ latitud Norte, $90^{\circ}$ 301 longitud Oeste para el período desde el año cero hasta el 1500 en el que fueron visibles 540 eclipses de sol y 1638 eclipses de luna.

\section{Discusión}

\section{¿Es un instrumento de predicción?}

La Tabla 2 muestra que hay pocas coincidencias con el catálogo de eclipses tomando las fechas en el códice como puntos de partida de los 69 intervalos (según la correlación GMT). Lo más notable es que no hay eclipses en las fechas iniciales. Esta es la principal razón que ha llevado a varios investigadores a suponer que la tabla es un instrumento para la predicción de eclipses, y que las fechas que aparecen en el códice, que son más de 500 años anteriores a la fecha en la que se piensa fue escrito el códice, son fechas que se obtuvieron restando un múltiplo del largo de la tabla a una fecha más contemporánea al manuscrito. Como se ve en la Tabla 2 unas fechas iniciales sirven más para predecir eclipses de sol, y la otra predice más eclipses de luna.

La Tabla 3 muestra que los intervalos de $176,177,178,147$ y 148 son los intervalos entre eclipses que más suceden a nivel global. La Figura 2 muestra que considerando la ventana de más menos un día se logran más de 50 coincidencias en promedio, con una distribución sesgada hacia valores de coincidencia más altos. Sin embargo su poder de predicción no es tan bueno a nivel local como lo muestra la Figura 3 con valores medios de 11 coincidencias de 69 para eclipses de sol, y 33 de 69 para eclipses de luna. Es notable que la tabla de eclipses del códice no contiene el intervalo de 30 días, que debería aparecer en promedio la mitad de veces que aparece el intervalo de 148 días. Esto es consistente con la idea (Bricker \& Bricker, 2011) de que las fechas al inicio de la tabla separadas por 30 días implican que la tabla se concentra en ventanas de 30 días alrededor de la fecha central dada por los 69 intervalos.

La tabla de eclipses en el códice es un buen instrumento de predicción a nivel global, tanto de eclipses de sol como de luna dependiendo si la fecha inicial es cercana a la luna nueva o la luna llena. Sin embargo la mayoría de eclipses no serían visibles en la región ocupada por la civilización maya. La justificación dada para esta idea es que no importaría mucho predecir un eclipse y que no ocurra, pero que ocurra un eclipse sin haber sido previsto sería una falta mayor. 
Tabla 4

Estadística de intervalos entre eclipses visibles en $18^{\circ}$ latitud Norte, $90.5^{\circ}$ longitud Oeste

\begin{tabular}{lll}
\hline Intervalo (días) & Eclipses de sol (\%) & Eclipses de luna (\%) \\
\hline 148 & 0.1855 & 0.061 \\
177 & 12.8 & 54.96 \\
$(354)$ & 12.614 & 14.64 \\
502 & 6.49 & 9.82 \\
$(531)$ & 7.6 & 2.87 \\
708 & 0.556 & 0.66 \\
$(857)$ & 5.57 & 3.23 \\
1,034 & 12.8 & 1.769 \\
1,211 & 9.646 & 0.24 \\
$(1,388)$ & 7.98 & 0.061 \\
1,565 & 6.3 & 0 \\
1,742 & 2.04 & 0 \\
\hline
\end{tabular}

Los intervalos entre paréntesis son notables por no aparecer en el códice, a pesar de tener un alto porcentaje.

Algunos investigadores concluyen que el hecho de que no hayan coincidencias con eclipses es una indicación de que la correlación entre calendarios GMT no aplica en el caso del códice (Böhm, Böhm, Klokočník, Vondrák, \& Kostelecký, 2012).

\section{¿Es un registro de eclipses observados?}

Es evidente que los 69 intervalos no pueden ser un registro de eclipses de sol, ya que no es posible observar tantos eclipses de sol desde una localidad en particular en un período de 33 años. No hay ninguna serie de eclipses de sol o de luna (visibles desde la región de estudio) en el período del año cero al 1500 que coincida con los 69 intervalos. Los datos de la Figura 3 muestran que el valor máximo de coincidencias vistas desde la región de interés para eclipses de sol es de 20 de 69, y para eclipses de luna es 44 de 69.
La distribución de los 69 intervalos en el códice no es típica de la distribución de intervalos entre eclipses observados en la región de estudio. Usando los porcentajes de la Tabla 4 se pueden calcular las probabilidades de aparición de cada intervalo en una distribución multinomial y compararlos con la distribución de 69 intervalos en el códice. En particular el intervalo de 148 días ocurre sólo una vez en los eclipses de sol o de luna en el período del año cero al 1500 por lo que es muy improbable que aparezca en una muestra de 69 intervalos, y en el códice aparece nueve veces. Además hay muchos otros intervalos con relativamente alta probabilidad que no aparecen en el códice. Esto implica que los 69 intervalos no son un registro de eclipses observados. Lo cual nos lleva a considerar los intervalos entre las 10 figuras.

La Tabla 4 muestra que los intervalos entre las 10 figuras sí corresponden al patrón de intervalos entre eclipses visibles desde la región de estudio. Vemos que los intervalos entre las 10 figuras en el códice aparecen para eclipses de luna y de sol, pero los intervalos de 
1,563 y de 1,742 días aparecen sólo en los eclipses de sol. Los intervalos de 1,034 y 1,211 días son más probables en los eclipses de sol que en eclipses de luna. Esto indica que la serie de eclipses asociada a las 10 figuras en el códice se parece más a la distribución de eclipses de sol vista desde la región de interés.

El hecho de que haya varias series de eclipses de luna que coinciden con los intervalos (Figura 4) se debe a que hay varios eclipses de luna intermedios que no tienen figura. Por ejemplo, nunca sucede que transcurran 1,563 o 1,742 días entre dos eclipses consecutivos de luna, pero puede que varios eclipses consecutivos tengan el primer y el último eclipse separados por 1,563 o 1,742 días. No hay una serie de eclipses de sol que coincida con las 10 figuras, la mayor cantidad de coincidencias es de siete en los datos de eclipses visibles desde las ciudades de Palenque, Piedras Negras, Yaxchilán y La Venta. Sin embargo hay 14 series de eclipses de luna en las que coinciden todas las figuras (y 98 series con nueve coincidencias), esto llevó a Verbelen (2006) a concluir que las 10 figuras se refieren a eclipses de luna. Estudiando el cielo durante estas series de eclipses encontró algunas similitudes entre el cielo y las figuras en una de estas series y propone una nueva constante de correlación en base a estas observaciones.

Otras razones a favor de que las figuras correspondan a eclipses de sol son que las fechas iniciales en la tabla corresponden mejor con eclipses de sol, que los dibujos contienen el glifo del sol, y que hay referencias a Venus en las figuras. Venus aparece siempre cerca del sol, y nunca se vería al mismo tiempo que un eclipse de luna. Tiene más sentido que hayan más eclipses aunque algunos no hayan sido observados y anotados como figuras, que pensar que un eclipse que no sucedió fuera anotado, lo cual hace pensar que no todas las figuras se refieren a eclipses de sol. Algunos investigadores opinan que no todas las figuras se refieren a eclipses, por ejemplo Lounsbury (1978) propone que la última figura en la página 58(b) donde aparece una representación de Venus se refiere a un evento anacrónico a la tabla. La inclusión de eventos anacrónicos complica la tarea de encontrar la coincidencia histórica. Otra circunstancia que complicaría todo es si los eclipses anotados fueron observados en distintos lugares, en vez de todos desde la misma localidad. Aún más compleja es la opinión de los Bricker y Bricker (2011), que consideran una versión extendida de la tabla que contiene los intervalos iniciales, y los múltiplos de la tabla, y proponen que algunas figuras no tienen sentido durante la primera pasada de la tabla, pero sí en alguno de los múltiplos siguientes.

\section{¿Es un registro de eclipses de sol, de luna o combinado?}

Las fechas dadas explícitamente por los 69 intervalos en la tabla de eclipses no pueden ser un registro combinado de eclipses de sol y luna ya que los intervalos son de cinco o seis lunaciones enteras, y entre eclipses de sol y luna deberían haber intervalos que son múltiplos impares de 15 días aproximadamente (el tiempo entre luna nueva y luna llena). La Tabla 2 ilustra esto explícitamente para las fechas de eclipses en el códice separadas por intervalos de 15 días. Unas fechas coinciden más con eclipses de sol y ningún eclipse de luna, mientras que otra fecha separada de estas por un intervalo de 15 días tiene más coincidencias con eclipses de luna pero ningún eclipse de sol. Esto también se comprobó mezclando los catálogos de eclipses de sol y de luna y buscando coincidencias en esta base de datos combinada. Como resultado no se halló ninguna serie nueva con eclipses combinados que coincida mejor con los intervalos en el códice.

Sin embargo cinco de las 10 figuras muestran un par de glifos de eclipse de sol y eclipse de luna juntos, apoyando la idea de que las fechas iniciales de la tabla separadas por 15 días llaman la atención sobre ventanas de tiempo que abarcan pares de eclipses de sol y de luna separados por 15 días.

\section{Conclusión}

Los intervalos entre las 10 figuras son intervalos que podemos encontrar en la distribución de eclipses de sol observados desde la región ocupada por la civilización maya (Tabla 4). Los datos de eclipses de sol visibles desde la región de estudio muestran un promedio de 11 coincidencias de eclipses de sol de un total de 69 en el período del año cero al 1500 (Figura 3 ), lo cual es consistente con que las 10 figuras hagan referencia a eclipses de sol. Ninguna serie de eclipses generada con los intervalos entre figuras tiene más de siete coincidencias con el catálogo de eclipses de sol visibles en la región de estudio en el período estudiado, por lo que no todas las figuras pueden ser asociadas a eclipses de sol.

Hay cinco figuras con glifos de eclipse de sol y eclipse de luna, tres figuras en las que no aparece en el texto el glifo de eclipse (aunque aparece en la figura en forma estilizada), y dos figuras en las que el glifo de eclipse de sol aparece en la columna anterior a la figura 
pero no en el texto que acompaña la figura, lo cual hace pensar que no todas las 10 figuras se refieren al mismo tipo de fenómeno.

La tabla es un buen instrumento para predecir eclipses a nivel global, pero no para predecir eclipses localmente ya que los 69 intervalos no corresponden a un registro de eclipses observados desde el área ocupada por la civilización maya. Las fechas dadas por los 69 intervalos corresponden a eclipses de sol, o de luna, pero no ambos. Dependiendo si la fecha inicial está cerca de la luna nueva o la luna llena. Sin embargo si se considera que las fechas separadas por 15 días al inicio de la tabla establecen una ventana de 30 días, es posible que algunas de las figuras se refieran a pares de eclipses separados por 15 días.

Tomando las fechas que aparecen en el códice según la correlación GMT no hay coincidencias con eclipses de sol o de luna, sin embargo, a lo largo de la historia han habido varias series de eclipses de luna que coinciden con los intervalos dados por las 10 figuras, $\mathrm{y}$ algunas series de eclipses de sol con seis coincidencias, y una serie de eclipses solares con siete coincidencias exactas. De forma que si las 10 figuras en la tabla son un registro histórico de eclipses, podría ser una de estas series con alta coincidencia, aunque es más probable que las fechas del códice están corridas por algún motivo que pensar que es la correlación entre calendarios la que hay que cambiar.

El esquema que surge es que los intervalos de 177 días son fechas en las que se espera que haya eclipse, y las 10 figuras insertadas dentro de la serie podrían referirse a eclipses de sol que si sucedieron, varios de los cuales son notables por ser pares de eclipses con un eclipse de luna sucediendo a un eclipse de sol unos 15 días después, aunque no todas las 10 figuras involucren eclipses de sol, ya que no hay series en el catálogo de eclipses vistos desde la región de estudio que coincidan con las 10 figuras.

Aunque la correlación GMT está bien establecida por varios medios, el hecho de que hayan series de eclipses de sol visibles desde varias ciudades mayas con seis o más coincidencias exactas con los intervalos entre las 10 figuras en la tabla, cerca de la época en la que se piensa fue escrito el códice, justifica un estudio más detallado del cielo durante estos eclipses para buscar alguna correlación con los detalles tan particulares que se muestran en las figuras.

\section{Agradecimientos}

Esta investigación fue cofinanciada por Digi-Usac-2017, Proyecto: 4.8.63.3.13.

\section{Referencias}

Aveni, A. (2001). Skywatchers of Ancient Mexico. Austin: University of Texas Press.

Böhm, V., Böhm, B., Klokočník, J., Vondrák, J., \& Kostelecký, J. (2012). Dating of Mayan calendar using long periodic astronomical phenomena in Dresden codex. Serbian Astronomy Journal (186)53-64 doi: 10.2298/SAJ121221001B

Bretagnon, P. (1982). Theory for the motion of all the planets - The VSOP82 solution. Astronomy \& Astrophysics, 114(2), 278-288.

Bricker, H., \& Bricker, V. (2011). Astronomy in the Maya codices. Philadelphia: American Philosophical Society.

Chapront-Touzé, M., \& Chapront, J. (1983). The lunar ephemeris ELP-2000. Astronomy \& Astrophysics, 124(1), 50-62.

Danjon, A. (1951). Les éclipses de lune para la pénombre en 1951. L'Astronomie, 65, 51-53.

Espenak, F., \& Meeus, J. (2006). Five millennium catalog of solar eclipses (NASA/TP-2006-214174. Hanover: NASA Center for AeroSpace Information.

Espenak, F., \& Meeus, J. (2009). Five millennium catalog of lunar eclipses (NASA/TP--2009-214173). Hanover: NASA Center for AeroSpace Information.

Forstemann, E. (1906). Commentary on the Maya manuscript in the Royal Public Library of Dresden. Cambridge: Harvard University Press.

Gates, W. (1932). The Dresden Codex. Baltimore: The Maya Society at the Johns Hopkins University.

Satterthwaite, L. S., \& Ralph, E. K. (1960). Radiocarbon dates and the Maya correlation problem. American Antiquity, 26, 165-18.

Lounsbury, F. (1978). Maya numeration, computation and calendrical Astronomy. Dictionary of Scientific Biography, 15, 759-818.

Macri, M., \& Vail, G. (2009). The new catalog of Maya hieroglyphs. The codical texts. Oklahoma: University of Oklahoma Press. 
Meinshausen, M. (1913). Über Sonnen- und mondfinsternisse in der Dresdener Mayahandschrift Zeitshrift für Ethnologie, 45, 221-227.

Milbrath, S. (1999). Star gods of the Maya. Astronomy in art, folklore, and calendars. Austin: University of Texas Press.
Thompson, J. E. (1972). A commentary on the Dresden Codex a maya hieroglyphic book.

Philadelphia: American Philosophical Society.

Verbelen, F. (2006). Eclipses and Supernova 1054 in the Dresden codex. Grimbergen: MIRA Observatory.

Willson, R. W. (1924) Astronomical notes on the Maya codices. Cambridge: Peabody Museum of American Archaeology and Ethnology. 